\title{
In vivo evaluation of atherosclerotic plaques and culprit lesions using noninvasive techniques
}

Federico Caobelli and Frank M. Bengel

Coronary angiography is the gold standard for assessing coronary artery disease (CAD) and vessel stenosis, but accumulating evidence has underscored that, at best, a weak correlation exists between the degree of stenosis and plaque rupture. Vulnerable plaque has, therefore, gained worldwide attention as a major determinant of the risk of developing symptomatic CAD. ${ }^{1}$ Given that coronary plaque inflammation has a pivotal role in the development of symptomatic $\mathrm{CAD},{ }^{2}$ functional imaging of this phenomenon might complement information on the anatomical extent of CAD. We read with great interest the Review by Tarkin et al. (PET imaging of inflammation in atherosclerosis. Nat. Rev. Cardiol. 11, 443-457; 2014), ${ }^{3}$ in which the authors describe noninvasive techniques, such as PET-CT with various tracers, to identify atherosclerotic plaques and stratify the risk of rupture. Such approaches are highly desirable for both disease prevention and to monitor treatment. These observations are commonly limited to large peripheral vessels, but conceptually, these techniques could be applied to the imaging of coronary arteries by capitalizing on the high spatial resolution and sensitivity of PET scanners.

The high spatial resolution afforded by PET has stimulated a variety of approaches to detect vessel inflammation using existing or novel radiotracers. Initial clinical evaluation using ${ }^{18} \mathrm{~F}$-fluoroxyglucose (FDG) showed promise. ${ }^{4}$ However, many subsequent studies have been focused on tracers with more specific targets, such as ${ }^{18}$ F-fluoride PET-CT, ${ }^{5}$ which can be used to track the presence of active calcification, correlated to macrophage infiltration, apoptosis, and necrosis, as seen in lesions at high risk of rupture. Additionally, the potential of somatostatin-receptor-binding $\beta$-emitters, such as ${ }^{68} \mathrm{Ga}$-DOTATATE, to identify active macrophage content in atherosclerotic plaques has been investigated. ${ }^{6}$

Unfortunately, despite the robust predictive value reported in patients with $\mathrm{CAD},{ }^{7}$ widespread uptake of PET imaging is hindered by high costs, low availability of PET scanners, and the short half-life of some PET tracers, requiring an on-site cyclotron and radiochemistry specialists, which limits its clinical application to specialized centres. By contrast, single-photon emission computed tomography (SPECT) cameras are more commonplace, which, coupled with the routine use of radioactive isotopes with a longer half-life than those used for PET, translates to enhanced availability. However, conventional SPECT hardware has limited spatial resolution, which impairs the delineation of vascular structures.

To overcome these limitations, new SPECT hardware has been introduced, optimized for cardiovascular imaging and equipped with cadmium-zinc-telluride (CZT) solid-state scintillators, which provide multiple advantages, such as high sensitivity, improved temporal and spatial resolution, and high energy resolution, enabling simultaneous detection of multiple radioisotopes within the energy spectrum. In particular, the higher spatial resolution $(6.7 \mathrm{~mm}$ versus $15.3 \mathrm{~mm}$ for central spatial resolution) and the improved sensitivity with CZT scintillators facilitate delineation of smaller differences in tracer uptake, allowing finer definition of vascular walls. ${ }^{8}$

Investigators have demonstrated a possible use of SPECT systems in the evaluation of atherosclerotic plaque. For example, ${ }^{99 \mathrm{~m} T c}$-labelling of autologous leukocytes allowed the infiltration of inflammatory cells to be visualized in atherosclerotic lesions. ${ }^{8}$ In this study, scintigraphy with radiolabelled leukocytes was used to detect inflamed vascular lesions, which correlated well with disease severity. ${ }^{9}$ In another study, the feasibility of using ${ }^{99 \mathrm{~m}} \mathrm{Tc}$-hydrazinonicotinamide (HYNIC)-interleukin-2 SPECT to track lymphocytic infiltration into carotid atherosclerotic lesions was examined. ${ }^{10}$

Capitalizing on the improved spatial resolution and sensitivity of CZT cameras might allow this technology to be applied to coronary plaque imaging, as is currently obtainable using PET-CT. Additional benefits arising from CZT cameras might include improved energy resolution, which would allow simultaneous evaluation of labelled lymphocytic infiltration together with tracers targeting other physiological processes, including apoptosis ( ${ }^{111}$ In-DTPA-annexin $)^{11}$ or angiogenesis ( ${ }^{111} \mathrm{In}$-RGD peptide). ${ }^{12}$ Moreover, the superior performance of CZT cameras might enable absolute quantification of plaque inflammation. CZT technology could also allow a reduction in the radiopharmaceutical dose that is administered, which would lower the radiation exposure to patients.

Studies involving larger cohorts of patients imaged with CZT SPECT than have been performed to date are warranted to assess whether vulnerable plaques can be identified with similar accuracy to that achieved using PET imaging. If the results of these trials are robust, the effect on clinical practice will be dramatic, facilitating the widespread functional evaluation of atherosclerotic plaques. Indeed, the deployment of new SPECT cameras might increase the applicability of this technique, to improve diagnostic accuracy and become a valuable tool in predicting prognosis and therapeutic outcome. Further studies are necessary to define the radiotracer binding thresholds that translate to physiologically relevant plaque inflammation, which would provide a valuable tool in risk stratification of patients. The widespread use of molecular imaging techniques will shift the clinical paradigm away from resultant anatomy towards causative physiology.

Klinik für Nuklearmedizin, Medizinische Hochschule Hannover, Carl Neuberg Straße, 1, 30625 Hannover, Germany (F.C., F.M.B.).

Correspondence to: F.C.

caobelli.federico@mh-hannover.de

Competing interests

F.C. receives a fellowship grant from Mallinckrodt. F.M.B. receives research grants from Mallinckrodt and Siemens, and speaker honoraria from Bayer, GE Healthcare, Mallinckrodt, and Siemens. 


\section{CORRESPONDENCE}

1. Fleg, J. L. et al. Detection of high-risk atherosclerotic plaque: report of the NHLBI Working Group on current status and future directions. JACC Cardiovasc. Imaging 5, 941-955 (2012).

2. Naghavi, M. et al. From vulnerable plaque to vulnerable patient: a call for new definitions and risk assessment strategies: part I. Circulation 108, 1664-1672 (2003).

3. Tarkin, J. M., Joshi, F. R. \& Rudd, J. H. F. PET imaging of inflammation in atherosclerosis. Nat. Rev. Cardiol. 11, 443-457 (2014).

4. Rudd, J. H. et al. ${ }^{18}$ Fluorodeoxyglucose positron emission tomography imaging of atherosclerotic plaque inflammation is highly reproducible: implications for atherosclerosis therapy trials. J. Am. Coll. Cardiol. 50, 892-896 (2007).
5. Joshi, N. V. et al. ${ }^{18} \mathrm{~F}$-fluoride positron emission tomography for identification of ruptured and high-risk coronary atherosclerotic plaques: a prospective clinical trial. Lancet $383,705-713$ (2014).

6. Schatka, I. et al. Peptide receptor-targeted radionuclide therapy alters inflammation in atherosclerotic plaques. J. Am. Coll. Cardiol. 62 2344-2345 (2013).

7. Tio, R. A. et al. Comparison between the prognostic value of left ventricular function and myocardial perfusion reserve in patients with ischemic heart disease. J. Nucl. Med. 50, 214-219 (2009).

8. Imbert, L. et al. Compared performance of highsensitivity cameras dedicated to myocardial perfusion SPECT: a comprehensive analysis of phantom and human images. J. Nucl. Med. 53, 1897-1903 (2012).
9. van der Valk, F. M. et al. In vivo imaging of enhanced leukocyte accumulation in atherosclerotic lesions in humans. J. Am. Coll. Cardiol. 64, 1019-1029 (2014).

10. Glaudemans, A. W. et al. In vivo and in vitro evidence that ${ }^{99} \mathrm{mTc}$-HYNIC-interleukin- 2 is able to detect $\mathrm{T}$ lymphocytes in vulnerable atherosclerotic plaques of the carotid artery. Eur. J. Nucl. Med. Mol. Imaging 41, 1710-1719 (2014).

11. Zhang, R. et al. Annexin A5-conjugated polymeric micelles for dual SPECT and optical detection of apoptosis. J. Nucl. Med. 52 , 958-964 (2011).

12. Terry, S. Y. et al. Imaging integrin $\alpha_{\mathrm{v}} \beta_{3}$ on blood vessels with ${ }^{111}$ In-RGD 2 in head and neck tumor xenografts. J. Nucl. Med. 55, 281-286 (2014). 